\title{
Immune correlates of metastatic melanoma patients treated with ipilimumab in combination with fotemustine in the phase II NIBIT-M1 study
}

\author{
Cristina Maccalli ${ }^{1,4^{*}}$, Hugues Nicolay ${ }^{2,4}$, Filippo Capocefalo ${ }^{1,4}$, Ester Fonsatti ${ }^{2,4}$, Carla Chiarucci ${ }^{2,4}$, Ornella Cutaia ${ }^{2,4}$, \\ Diana Giannarelli, ${ }^{3,4}$, Giorgio Parmiani ${ }^{1,4}$, Anna Maria Di Giacomo ${ }^{2,4}$, Michele Maio ${ }^{2,4}$
}

From Society for Immunotherapy of Cancer 28th Annual Meeting

National Harbor, MD, USA. 8-10 November 2013

\section{Background}

Ipilimumab (IPI) in combination with fotemustine (FTM) has shown a promising clinical activity in metastatic melanoma (MM) patients (pts) enrolled in the NIBIT-M1 trial (Di Giacomo, et al., Lancet Oncology, 2012). This study investigated changes in immunological parameters in the course of treatment.

\section{Material and methods}

MM pts received an induction therapy with IPI $10 \mathrm{mg} / \mathrm{kg}$ every 3 weeks (Q3W) for four doses and FTM $100 \mathrm{mg} / \mathrm{m} 2$ weekly for 3 weeks. Peripheral blood lymphocytes (PBMC) and sera were collected at baseline, wk12, and wk24 to perform phenotypic and functional $\mathrm{T}$ cell assays, and to investigate humoral responses against a panel of tumorassociated antigens (TAAs) and soluble NKG2D ligands (sNKG2DL).

\section{Results}

Circulating central memory $\mathrm{T}(\mathrm{Tcm})$ cell populations, both CD4+ and CD8+, co-expressing CD45RO, CD27, CD28, CCR7, CD62L, were increased following treatment both at wk12 and wk24. Interestingly, T cells coexpressing CD4, BTLA and CD45RO were increased in pts with clinical benefit, while CD8+ Tcm co-expressing BTLA were augmented in pts with objective responses. Circulating T cells reactive against NY-ESO-1, MART-1, gp100 and TYRP-1, were found in 12/23 pts expressing at least one of the HLA-A1, A2, -A3 or A24 alleles with induction or augmentation of TAA reactivity in the course of treatment. Moreover, at least one sNKG2DL

'Unit of Immuno-biotherapy of Melanoma and Solid Tumors, Fondazione Centro San Raffaele, Milan, Italy

Full list of author information is available at the end of the article was found, though heterogeneously, in sera of $26 / 38$ pts. Of note, in 10 pts, down-modulation of at least $2 / 4$ investigated sNKG2DLs were detectable in relation with TAAspecific responses. Finally, at baseline, antibodies against NY-ESO-1, MAGE-A3, SSX-2, HMW-MAA, TYRP-1 and MSLN were found in sera of 9-15-8-7-7-6 of 40 investigated MM pts, respectively. Substantial changes were seen during therapy showing induced humoral responses against at least one investigated TAA in 10/40 pts at wk12 and/or $w k 24$. In addition, up-regulation, equivalent to at least twofold of the pre-existing antibody levels, against at least one TAA was detected in 5/40 pts at wk12 and/or wk24.

\section{Conclusions}

Our results, although preliminary, indicate that IPI in combination with FTM in MM pts induced changes in circulating $\mathrm{T}$ subpopulations and in their TAA-specific responses as well as in circulating antibodies to selected TAAs probably contributing to the observed clinical activity.

\section{Authors' details}

'Unit of Immuno-biotherapy of Melanoma and Solid Tumors, Fondazione Centro San Raffaele, Milan, Italy. ${ }^{2}$ Division of Medical Oncology and

Immunotherapy, Azienda Ospedaliera Universitaria, Senese, Istituto Toscano Tumori, Siena, Italy. ${ }^{3}$ Statistics, Regina Elena National Cancer Institute, Rome, Italy. ${ }^{4}$ On behalf of the NIBIT-M1 Investigators group, Siena, Italy.

Published: 7 November 2013

doi:10.1186/2051-1426-1-S1-P107

Cite this article as: Maccalli et al: Immune correlates of metastatic melanoma patients treated with ipilimumab in combination with

fotemustine in the phase II NIBIT-M1 study. Journal for ImmunoTherapy of Cancer 2013 1(Suppl 1):P107. 